\title{
Le chanvre (Cannabis sativa L.) et sa réglementation Européenne en 2003. Teneur en $\Delta$-9-THC des variétés cultivées en France
}

\section{Hemp (Cannabis sativa L.) and its European regulation in 2003. $\Delta-9-T H C$ level of varieties cultivated in France}

\section{Gilbert FOURNIER}

Laboratoire de Pharmacognosie, UMR 8076, CNRS (BioCIS), Faculté de Pharmacie, 5, rue J.-B. Clément - 92296 CHÂTENAY-MALABRY

Tél : 0146835597 - Fax : 0146835710 - E-mail : gilbert.fournier@cep.u-psud.fr

(Reçu le 31 mars 2003 ; accepté le 17 septembre 2003)

\section{RÉ SUMÉ}

Selon la réglementation européenne, depuis 2001, $30 \%$ des superficies de chanvre destiné à la production de fibres doivent être contrôlées. Le dosage du $\Delta$-9-tétrahydrocannabinol $(\Delta-9-T H C)$ dans les différentes variétés de chanvre doit être réalisé et la teneur ne doit pas être supérieure à $0,2 \%$. En 2001, 278 prélèvements ont ainsi été effectués, et 274 en 2002. Comme cela avait déjà été constaté lors de précédentes études, les teneurs en $\Delta-9-T H C$ de toutes les cultures françaises sont inférieures à $0,2 \%$ et respectent ainsi la norme en vigueur.

\section{MOTS CLÉS}

Chanvre, Cannabis sativa, delta-9-tétrahydrocannabinol, dosage, réglementation.

\section{SUMMARY}

According to the European regulation, since 2001, $30 \%$ of hemp areas intended for fibre production must be inspected. The level of $\Delta-9$-tetrahydrocannabinol $(\Delta-9-T H C)$ contained in the different hemp varieties has to be determined and must not exceed $0.2 \%$. In 2001, 278 samples were analyzed, and 274 in 2002. As it was observed in previous studies, the $\Delta-9$ THC level in all French cultivation is lower than $0.2 \%$, so is in accordance with the current norm.

\section{KEY-WORDS}

Hemp, Cannabis sativa, delta-9-tetrahydrocannabinol, quantification, regulation. 


\section{Introduction}

La culture du chanvre (Cannabis sativa L., Cannabaceae) est traditionnelle en France. Elle se fait encore aujourd'hui sur plus de 7000 ha. Les fibres de la tige ont des utilisations industrielles variées. La principale substance responsable des propriétés psychoactives de la plante est le $\Delta$-9-tétrahydrocannabinol ( $\Delta$-9THC). Des opérations de sélection, menées depuis une vingtaine d'années, ont permis d'obtenir des nouvelles variétés à teneur particulièrement faible en $\triangle$-9-THC $(1,2)$. Réglementairement, jusqu'en 2000 , cette teneur ne devait pas être supérieure à $0,3 \%$. Depuis, le protocole d'échantillonnage a été modifié et le seuil réglementaire relatif à la teneur en $\Delta-9$-THC a été abaissé à $0,2 \%(3,4)$. Après avoir rappelé la réglementation en vigueur, profondément remaniée depuis l'année 2000, le présent travail est consacré à l'étude des résultats des contrôles officiels effectués sur les variétés de chanvre cultivées en France en 2001 et en 2002.

\section{Rappels de la réglementa- tion en vigueur \\ Textes du Code de la Santé Publique}

\section{1 - Article R. 5181}

Le décret $n^{\circ} 88-1231$ du 29 décembre 1988 (5), stipule dans son article $1^{\text {er }}$ que : $\ll$ Sont interdits la production, la mise sur le marché, l'emploi et l'usage $1^{\circ}$ du cannabis, de sa plante et de sa résine, des préparations qui en contiennent ou de celles qui sont obtenues à partir du cannabis, de sa plante ou de sa résine ; $2^{\circ}$ des tétrahydrocannabinols, de leurs esters, éthers, sels ainsi que des sels des dérivés précités et de leurs préparations». Il précise cependant que : "des dérogations aux dispositions énoncées ci-dessus peuvent être accordées par le ministre chargé de la santé, aux fins de recherche et de contrôle ainsi que de fabrication de dérivés autorisés; cependant, les ministres chargés de la santé, de l'agriculture, de l'industrie et des douanes peuvent, par arrêté conjoint, autoriser la culture, l'importation, l'exportation et l'utilisation industrielle et commerciale de variétés de cannabis dépourvues de propriétés stupéfiantes».

\section{2 - Application de l'article R. 5181}

L'arrêté du 22 août 1990 porte application de cet article du Code de la Santé Publique (6) et autorise certaines cultures : «sont autorisées au sens de l'article R. 5181 du code susvisé, la culture, l'importation, l'exportation, l'utilisation industrielle et commerciale-(fibres et graines) des variétés de Cannabis sativa $\mathrm{L}$. répondant aux critères suivants : le poids de THC (tétrahydrocan- nabinols) de ces variétés par rapport au poids d'un échantillon porté à poids constant n'est pas supérieur à 0,30 p. 100 ; la détermination du taux de tétrahydrocannabinols et la prise d'échantillons en vue de cette détermination sont effectuées selon la méthode unique prévue en annexe I (Méthode communautaire pour la détermination quantitative du $\Delta-9-$ THC des variétés de chanvre). Les variétés autorisées sont les suivantes : Carmagnola, C.S., Delta-Llosa, Delta-405, Fedora 19, Fedrina 74, Felina 34, Ferimon, Fibranova, Fibrimon 24, Fibrimon 56, Futura». Depuis la parution de ce texte, deux nouveaux arrêtés complètent la liste des variétés de Cannabis sativa L. autorisées : Epsilon 68 et Santhica 23 (Arrêté du 27 mai 1997) (7) ; Fedora 17, Felina 32, Futura 75 et Dioïca 88 (Arrêté du 2 juillet 1999) (8).

Pour le Code de la Santé Publique, seuls sont donc autorisés la production, la mise sur le marché, l'emploi et l'usage de certaines variétés de Cannabis sativa dont la teneur en $\Delta-9$-THC n'est pas supérieure à $0,3 \%$. Cette teneur est déterminée selon une méthode standardisée.

\section{Autres textes officiels}

Depuis l'année 2000, plusieurs règlements concernant le chanvre sont parus au Journal officiel des Communautés européennes.

\section{1 - Règlement (CE) $n^{\circ}$ 1177/2000}

Le règlement $(\mathrm{CE}) \mathrm{n}^{\circ} 1177 / 2000$ de la Commission du 31 mai 2000 modifiant le règlement (CEE) $n^{\circ} 1164 / 89$ relatif aux modalités d'aide concernant le lin textile et le chanvre (9) indique que l'annexe $\mathrm{C}$ du règlement (CEE) $n^{\circ} 1164 / 89$ est remplacée par une nouvelle annexe décrivant précisément la méthode communautaire pour la détermination quantitative du $\Delta-9-\mathrm{THC}$ des variétés de chanvre.

\section{2 - Règlement (CE) $n^{\circ}$ 1672/2000}

Le règlement $(\mathrm{CE}) \mathrm{n}^{\circ} \cdot 1672 / 2000 \mathrm{du}$ Conseil du 27 juillèt 2000 modifiant le règlement $(\mathrm{CE}) \mathrm{n}^{\circ} 1251 / 1999$ institue un régime de soutien aux producteurs de certaines cultures arables, pour $y$ inclure le lin et le chanvre destinés à la production de fibres (10). Les motivations du Conseil de l'Union Européenne étaient les suivantes : "Afin d'éviter des risques de détournement des objectifs visés par le règlement $(\mathrm{CE}) \mathrm{n}^{\circ}$ 1251/1999, il est nécessaire de subordonner l'octroi du paiement à l'hectare pour le lin et le chanvre destinés à la production de fibres à certaines conditions de culture. Dans le cas du chanvre, il convient de prévoir des mesures spécifiques afin d'éviter que des cultures illicites se cachent parmi celles qui peuvent bénéficier des paiements à la surface et perturbent ainsi l'organisation 
commune de marché de ce produit. Il est donc nécessaire de prévoir que ces paiements ne sont octroyés que pour les superficies où ont été utilisées des variétés de chanvre offrant certaines garanties en ce qui concerne la teneur en substances psychotropes». Les règles sont décrites dans l'article 5 bis : «Pour le lin et le chanvre destinés à la production de fibres, le paiement à la surface est subordonné, selon les cas, à la conclusion d'un des contrats ou au dépôt de l'engagement, visés à l'article 2, paragraphe 1 , du règlement $(\mathrm{CE}) \mathrm{n}^{\circ} 1673 / 2000$. Pour le chanvre destiné à la production de fibres, le paiement à la surface est également subordonné à l'utilisation de variétés dont la teneur en tétrahydrocannabinol n'est pas supérieure à $0,2 \%$. Les Etats membres instaurent un système de contrôle du contenu en tétrahydrocannabinol sur au moins $30 \%$ des superficies de chanvre destiné à la production de fibres faisant l'objet de demande de paiement. Toutefois, dans le cas où l'Etat membre établit un système d'autorisation préalable de ladite culture, le pourcentage minimal est de $20 \%$ \%.

\section{3 - Règlement $(\mathrm{CE}) \mathrm{n}^{\circ} \mathbf{2 8 6 0 / 2 0 0 0}$}

Le règlement $(\mathrm{CE}) \mathrm{n}^{\circ} 2860 / 2000$ de la Commission du 27 décembre 2000 modifiant le règlement (CE) $n^{\circ}$ 2316/1999 portant modalités d'application du règlement (CE) $n^{\circ} 1251 / 1999$ du Conseil instituant un régime de soutien aux producteurs de certaines cultures arables pour y inclure le lin et le chanvre destinés à la production de fibres, préciser les règles concernant les superficies gelées et modifier les superficies de base pour la Grèce et le Portugal (11). «L'article 5 bis du règlement (CE) 1251/1999 prévoit, d'une part, l'utilisation de variétés dont la teneur en tétrahydrocannabinol n'est pas supérieure à $0,2 \%$ et, d'autre part, l'établissement par les Etats membres d'un système de contrôle du contenu en tétrahydrocannabinol du chanvre. Pour permettre la réalisation de ce contrôle, il y a lieu de prévoir des mesures spécifiques, notamment le maintien de la culture de chanvre jusqu'à une certaine date ; ... dans le cas du chanvre, la teneur en tétrahydrocannabinol des variétés admises ne peut être supérieure à $0,2 \%$. Il est donc nécessaire d'établir une liste de variétés éligibles. Pour faciliter le passage du régime en vigueur à celui établi par le règlement (CE) $\mathrm{n}^{\circ} 1251 / 1999$, il y a lieu de prévoir aussi une liste de variétés de chanvre admises temporairement pour la campagne 2001/2002 qui devront faire l'objet d'analyses complémentaires au cours de la campagne 2001/2002. Pour renforcer la garantie, il convient de prévoir que les semences utilisées soient certifiées ; ... il est nécessaire d'établir la méthode à utiliser pour la détermination quantitative du tétrahydrocannabinol du chanvre destiné à la production de fibres et de prévoir une communication à la Commission des résultats des analyses effectuées conformément à cette méthode ; ... les Etats membres contrôlent $30 \%$ des superficies faisant l'objet d'une demande de paiement pour le chanvre destiné à la production de fibres et $20 \%$ en cas de système d'autorisation préalable de ladite culture. Il apparaît nécessaire de préciser les exigences liées à ces contrôles».

Le règlement arrêté est donc le suivant : «... le chanvre destiné à la production de fibres, pour permettre les contrôles prévus à l'article 5 bis, paragraphe 2, du règlement $(\mathrm{CE}) \mathrm{n}^{\circ} 1251 / 1999$, la culture doit être entretenue, dans des conditions de croissance normale, conformément aux normes locales, au moins jusqu'à dix jours après la fin de la floraison. Toutefois, l'Etat membre peut autoriser la récolte de chanvre destiné à la production de fibres après le début de la floraison, mais avant l'échéance des dix jours après la fin de la floraison, si le producteur en question a déjà fait l'objet du contrôle prévu à l'article 5 bis du règlement (CE) $1251 / 1999$, ou si tous les contrôles à effectuer ... ont été accomplis».

Il est en outre précisé que : «Pour l'application de l'article 5 bis, paragraphe 2 , du règlement $(C E) \mathrm{n}^{\circ}$ 1251/1999, la méthode à utiliser par les autorités compétentes de l'Etat membre pour procéder à la constatation du taux de tétrahydrocannabinol (THC) sur un pourcentage des superficies ensemencées en chanvre destiné à la production de fibres faisant l'objet de demandes de paiement est décrite à l'annexe XIII. Les Etats membres transmettent à la Commission, au plus tard le 15 novembre de la campagne de commercialisation en cause, un rapport sur les constatations du taux de THC effectuées. Ce rapport comporte, par variété, notamment : a) pour la procédure $A$, l'indication du moment où le prélèvement a été effectué ; b) le nombre de tests effectués ; c) les résultats obtenus par niveaux de THC, échelonnés par gradation de $0,1 \%$; d) les mesures prises au niveau national».

«Dans le cas où les constatations effectuées montrent, pour un nombre significatif d'échantillons d'une variété donnée, des teneurs en THC supérieures à la limite prévue à l'article 5 bis, paragraphe 1 , deuxième alinéa, du règlement $(C E) n^{\circ} 1251 / 1999$, le Commission peut, sans préjudice d'autres mesures, décider, selon la procédure prévue à l'article 23 du règlement $(\mathrm{CEE}) \mathrm{n}^{\circ}$ $1766 / 92$, de recourir, au cours de la campagne suivante, à la procédure $\mathrm{B}$ pour la variété concernée. Les variétés de chanvre destiné à la production de fibres figurant au point $2 \mathrm{~b}$ ) de l'annexe XII du présent règlement font l'objet de la procédure B au cours de la campagne 2001/2002 dans tous les Etats membres où elle sont cultivées». 
Enfin, «le contrôle du contenu en THC sur au moins $30 \%$ des superficies de chanvre destiné à la production de fibres faisant l'objet d'une demande de paiement doit porter sur au moins $30 \%$ des demandes concernées et sur toutes les variétés de semences utilisées».

Les variétés de chanvre destiné à la production de fibres admises au bénéfice du régime de soutien sont listées dans l'annexe XII : Carmagnola, Cs, Dioica 88, Epsilon 68, Fedora 17, Fédrina 74, Felina 32, Felina 34, Ferimon, Fibranova, Fibrimon 24, Fibrimon 56, Futura, Futura 75 , Santhica 23. Les variétés de chanvre destiné à la production de fibres admises pour la campagne 2001/2002 sont les suivantes : Beniko, Bialobrzeskie, Delta 405, Fasamo, Fedora 19, Juso 14, Kompolti, Uso 31.

\section{4 - Règlement (CE) n ${ }^{\circ}$ 327/2002}

Le règlement $(\mathrm{CE}) \mathrm{n}^{\circ} 327 / 2002$ de la Commission du 21 février 2002 modifiant le règlement (CEE) $n^{\circ}$ 2316/1999 portant modalités d'application du règlement (CE) $n^{\circ} 1251 / 1999$ du Conseil instituant un régime de soutien aux producteurs de certaines cultures arables (12) précise que : «L'article 5 bis, paragraphe 2 , du règlement $(\mathrm{CE}) \mathrm{n}^{\circ} 1251 / 1999$ prévoit que les Etats membres contrôlent au moins $30 \%$ des superficies faisant l'objet d'une demande de paiement pour le chanvre destiné à la production de fibres et $20 \%$ en cas de système d'autorisation préalable de ladite culture. Sur la base de l'expérience acquise au cours de la campagne 2001/2002, il apparaît nécessaire de préciser audelà de ce qui est prévu dans le règlement $(\mathrm{CE}) \mathrm{n}^{\circ}$ 2316/1999 les exigences liées à ces contrôles».

«Les Etats membres ont communiqué les résultats d'analyse du taux en tétrahydrocannabinol des variétés de chanvre ensemencées en 2001. Il convient de tenir compte de ces résultats pour l'établissement de la liste des variétés qui peuvent bénéficier au cours des prochaines campagnes des paiements à la surface et la liste des variétés de chanvre admises temporairement pour la campagne 2002/2003 qui devront faire l'objet d'analyses complémentaires au cours de cette campagne».

Les variétés de chanvre destiné à la production de fibres admises au bénéfice du régime de soutien sont données dans l'annexe XII : Carmagnola, Beniko, Cs, Deltallosa, Delta 405, Dioica 88, Epsilon 68, Fedora 17, Fédrina 74, Felina 32, Felina 34, Ferimon, Fibranova, Fibrimon 24, Fibrimon 56, Futura, Futura 75, Juso 14, Santhica 23, Uso 31 . Les variétés de chanvre destiné à la production de fibres admises pour la campagne 2002/2003 sont les suivantes : Bialobrzeskie, Fasamo, Fedora 19, Santhica 27.

\section{5 - Règlement (CE) $n^{\circ} 335 / 2003$}

Enfin, en 2003, le règlement (CE) $n^{\circ} 335 / 2003$ de la
Commission du 21 février 2003 modifiant le règlement (CEE) $n^{\circ} 2316 / 1999$ portant modalités d'application du règlement $(\mathrm{CE}) \mathrm{n}^{\circ} 1251 / 1999 \mathrm{du}$ Conseil instituant un régime de soutien aux producteurs de certaines cultures arables (13) précise que :

«Les Etats membres ont communiqué les résultats d'analyse du taux en tétrahydrocannabinol des variétés de chanvre ensemencées en 2002. Il convient de tenir compte de ces résultats pour l'établissement de la liste des variétés qui peuvent bénéficier au cours des prochaines campagnes des paiements à la surface et la liste des variétés de chanvre admises temporairement pour la campagne 2003/2004 qui devront faire l'objet d'analyses complémentaires au cours de cette campagne ; les variétés de chanvre destiné à la production de fibres admises au bénéfice du régime de soutien sont données dans l'annexe XII : Carmagnola, Beniko, Chameleon, Cs, Delta-llosa, Dioica 88, Epsilon 68, Fedora 17, Felina 32, Felina 34, Ferimon, Fibranova, Fibrimon 24, Futura 75, Juso 14, Red Petiole, Santhica 23, Uso 31. Celles destinées à la production de fibres admises pour la campagne 2003/2004 sont : Delta 405, Bialobrzeskie, Fasamo, Fedora 19, Fédrina 74, Fibrimon 56, Futura, Santhica 27».

En conclusion, selon la réglementation européenne en vigueur, les cultures de certaines variétés de chanvre peuvent bénéficier d'un régime de soutien. Leur teneur en $\Delta-9-\mathrm{THC}$ ne doit pas être supérieure à $0,2 \%$. La réglementation relative au chanvre est donc complexe. Deux séries de textes coexistent : articles du Code de la Santé publique français d'une part et règlements parus au Journal officiel des Communautés européennes d'autre part. Une harmonisation de cette réglementation est en cours. Quoi qu'il en soit et en tenant compte de ces différentes réglementations, la production, la mise sur le marché, l'emploi et l'usage du cannabis sont interdits. Cependant, pour une utilisation industrielle et commerciale, certaines variétés de Cannabis dépourvues de propriétés stupéfiantes peuvent être cultivées, sur autorisations ministérielles. Seule est ainsi aujourd'hui autorisée en France la culture de 18 variétés (Code de la Santé Publique) ; mais, paradoxalement, en 2003, 26 variétés auraient pu bénéficier de paiements à la surface de la part de la CEE (Tableau I). Depuis 2001, chaque année, $30 \%$ des superficies de chanvre destinées à la production de fibres doivent être contrôlées ( $20 \%$ en cas d'autorisation préalable de la culture). Les prélèvements et le dosage du $\Delta-9$-THC doivent être réalisés selon un protocole normalisé. La teneur en $\Delta-9$-THC ne doit pas dépasser $0,2 \%$. Les résultats des analyses effectuées en 2001 et en 2002 sont présentés ci-après. 


\section{Méthodologie}

Les dosages du $\Delta-9-$ THC ont été effectués, selon la procédure officielle (annexe $\mathrm{C}$ du règlement $(\mathrm{CE}) \mathrm{n}^{\circ}$ 1177/2000 de la Commission du 31 mai 2000), sur les variétés mentionnées dans le tableau I.

Tableau I : Variétés de chanvre dont la culture est autorisée selon le Code de la Santé Publique (CSP) et qui peuvent bénéficier d'une aide de la part de la CEE.

\begin{tabular}{|l|c|c|c|c|}
\hline \multirow{2}{*}{ Variétes autorisées } & CSP & \multicolumn{3}{|c|}{ CEE } \\
\cline { 3 - 5 } & & 2001 & 2002 & 2003 \\
\hline Beniko & & + & + & + \\
\hline Bialobrzeskie & & + & + & + \\
\hline Carmagnola & + & + & + & + \\
\hline Chameleon & & & & + \\
\hline CS & + & + & + & + \\
\hline Delta 405 & + & + & + & + \\
\hline Delta Llosa & + & & + & + \\
\hline Diö́ca 88 & + & + & + & + \\
\hline Epsilon 68 & + & + & + & + \\
\hline Fasamo & & + & + & + \\
\hline Fedora 17 & + & + & + & + \\
\hline Fedora 19 & + & + & + & + \\
\hline Fedrina 74 & + & + & + & + \\
\hline Felina 32 & + & + & + & + \\
\hline Felina 34 & + & + & + & + \\
\hline Ferimon & + & + & + & + \\
\hline Fibranova & + & + & + & + \\
\hline Fibrimon 24 & + & + & + & + \\
\hline Fibrimon 56 & + & + & + & + \\
\hline Futura & + & + & + & + \\
\hline Futura 75 & + & + & + & + \\
\hline Juso 14 & & + & + & + \\
\hline Kompolti & & + & & \\
\hline Red Petiole & & & & + \\
\hline Santhica 23 & + & + & + & + \\
\hline Santhica 27 & & & + & + \\
\hline Uso 31 & & + & + & + \\
\hline
\end{tabular}

\section{Échantillonnage}

Dans chaque parcelle, les prélèvements, effectués pendant la journée, concernent les 30 centimètres supérieurs de 50 plantes, au cours d'une période comprise entre les vingt jours après le début et les dix jours après la fin de la floraison. Le séchage est effectué à une température inférieure à $70^{\circ} \mathrm{C}$. Les échantillons secs sont débarrassés des tiges et des graines puis broyés (poudre demi-fine).

\section{Analyse du contenu en $\Delta-9-$ THC :}

Les réactifs sont le $\Delta-9$-THC et le squalène chromatographiquement purs. Le squalène est l'étalon interne, en solution à $35 \mathrm{mg} / 100 \mathrm{ml}$ d'hexane. L'extraction du $\Delta-9$-THC se fait de la façon suivante : dans un tube, peser $100 \mathrm{mg}$ de la poudre préparée ci-dessus et extraire par $5 \mathrm{ml}$ de solution d'extraction. Placer dans un bac à ultrasons pendant 20 min. Centrifuger. Prélever le soluté surnageant, qui est la solution à chromatographier. Injecter $1 \mu$.

L'appareillage est un chromatographe en phase gazeuse muni d'un détecteur à ionisation de flamme et injecteur split/splitless. La séparation se fait sur une colonne capillaire en verre de $25 \mathrm{~m}$ de long et $0,22 \mathrm{~mm}$ de diamètre (phase apolaire de type $5 \%$ phényl-méthylsiloxane). La température du four est de $260^{\circ} \mathrm{C}$.

La gamme d'étalonnage comporte quatre points : 0,04 $-0,10-0,25$ et $0,50 \mathrm{mg} / \mathrm{ml}$ de $\Delta-9-\mathrm{THC}$ en solution d'extraction.

Le résultat est exprimé en grammes de $\Delta-9-\mathrm{THC}$ pour 100 grammes d'échantillon d'analyse, séché jusqu'à poids constant. Le résultat est affecté d'une tolérance de $0,03 \%$ en valeur absolue (9).

\section{Résultats et discussion}

La figure 1 rassemble, pour 2001 et 2002, les moyennes des teneurs en $\Delta-9$-THC de toutes les variétés cultivées. Sont également reportées les teneurs minimales et maximales constatées.

Les moyennes des teneurs en $\Delta-9$-THC de la plupart des variétés cultivées en France en 2001 et en 2002 sont inférieures à $0,1 \%$ (rappel : la norme est l'utilisation de variétés dont la teneur en tétrahydrocannabinol n'est pas supérieure à $0,2 \%$ ). Il n'y a qu'une variété ayant une teneur moyenne supérieure à $0,1 \%$; il s'agit de la variété Juso 14, cultivée en 2001 et pour laquelle il n'y a qu'un seul résultat. Cette variété, bien que conforme à la législation en vigueur, n'a pas été cultivée en France en 2002. En ce qui concerne les valeurs maximales constatées pour chacune de ces variétés, aucune ne dépasse $0,2 \%$.

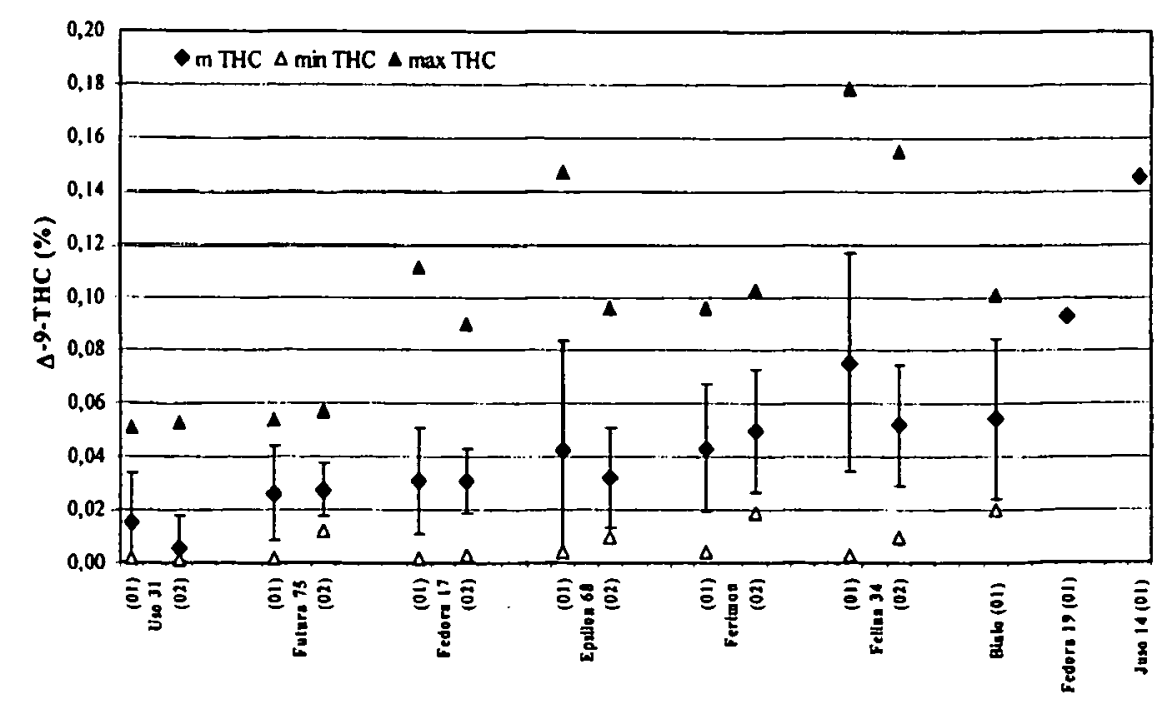

Figure 1 : Teneur en $\Delta-9-T H C$ du chanvre destiné à la production de fibres cultivé en France en 2001 et en 2002 $(m$ THC $=$ teneur moyenne en $\triangle-9-T H C ; \min$ et $\max T H C=$ teneur minimale et teneur maximale en $\Delta-9-T H C$ ). 
Les résultats des dosages du $\Delta$-9-THC montrent une variabilité plus ou moins importante selon les variétés cultivées. Cette variabilité est matérialisée, sur la figure 1, par les «barres d'erreurs» (écart-types) relatives aux moyennes calculées pour chaque variété.

Pour mieux visualiser cette variabilité, sont ici détaillés, à titre d'exemple, les résultats des analyses 2001 et 2002 sur les deux variétés les plus cultivées en France (cf. Tableau II) : Fedora 17 (Figure 2) et Felina 34 (Figure 3). La première est commercialisée depuis 2000 , la seconde depuis 1987 . Les résultats ici présentés pour 2001 et 2002 confirment en tous points (teneurs moyennes, valeurs maximales, variabilité) les constatations qui avaient été faites lors d'une récente étude (4). Celle-ci avait concerné les dix variétés de chanvre à fibres cultivées en France entre 1987 et 2001. Les teneurs en $\triangle$-9-THC de ces variétés n'ont jamais dépassé la norme en vigueur.

Tableau II : Variétés cultivées et nombre d'échantillons prélevés dans les cultures de chanvre en France en 2001 et en 2002.

\begin{tabular}{|c|l|c|}
\hline Année & Variétés cultivées & Nombre d'échantillons prélevés \\
\hline $\mathbf{2 0 0 1}$ & Uso 31 & 11 \\
& Futura 75 & 9 \\
& Federa 17 & 107 \\
& Epsilon 68 & 10 \\
& Ferimon & 28 \\
& Felina 34 & 106 \\
& Bialobrzeskie & 5 \\
& Fedora 19 & 1 \\
& Juso 14 & 1 \\
\hline $\mathbf{2 0 0 2}$ & Uso 31 & 18 \\
& Futura 75 & 20 \\
& Fedora 17 & 96 \\
& Epsilon 68 & 26 \\
& Ferimon & 23 \\
& Felina 34 & 91 \\
\hline
\end{tabular}

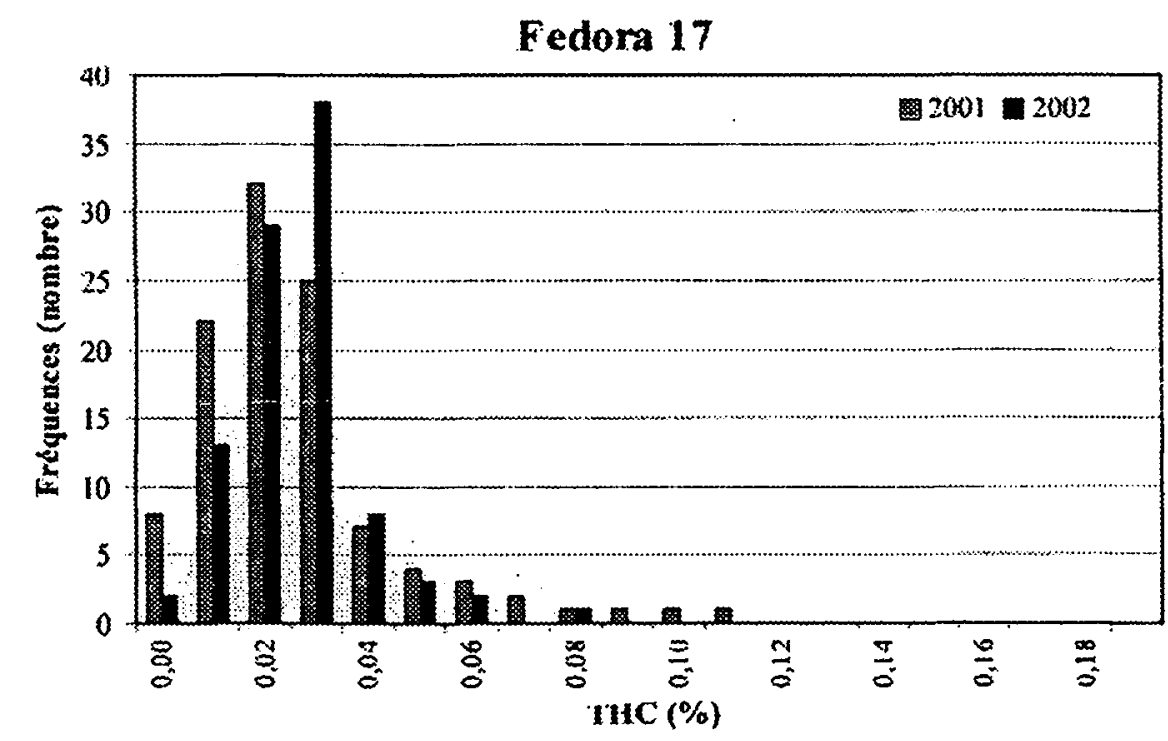

Figure 2 : Variations de la teneur en $\Delta-9-T H C$ de la variété Fedora 17 cultivée en France en 2001 et en 2002.
Felina 34

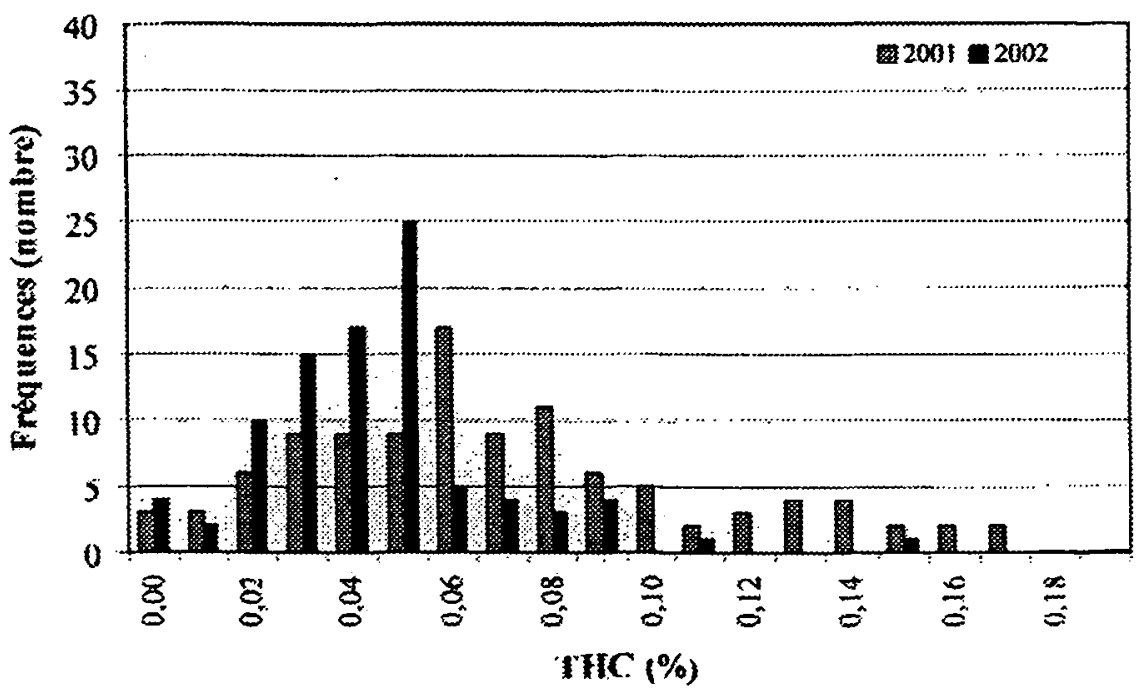

Figure 3 : Variations de la teneur en $\Delta-9-T H C$ de la variété Felina 34 cultivée en France en 2001 et en 2002.

\section{Conclusion}

Depuis plus de vingt ans, le chanvre est cultivé en France chaque année sur plus de 7000 hectares. De telles superficies représentent près de la moitié de la production européenne de cette plante. Les travaux de sélection entrepris dans notre pays depuis de nombreuses années ont eu, notamment, comme objectif d'abaisser la teneur en $\Delta-9$-THC pour que de telles cultures ne puissent constituer un quelconque danger en terme de santé publique. Ainsi la teneur en $\Delta-9-T H C$ ne doit pas excéder $0,2 \%$.

Selon la réglementation, en vigueur depuis 2001, les contrôles doivent être réalisés sur $30 \%$ des cultures. En 2001, 9 variétés ont été cultivées et 278 prélèvements ont été analysés ; en 2002, 6 variétés seulement ont été cultivées et 274 prélèvements ont été effectués. La détermination de la teneur en $\Delta-9$-THC sur tous ces prélèvements a permis de confirmer que toutes les variétés de chanvre cultivées en France en 2001 et 2002 ont respecté la norme en vigueur quant à leur teneur en $\Delta-9$-THC $(\leq 0,2 \%)$.

Remerciements à l'Office National Interprofessionnel des Céréales (O.N.I.C.) et à l'Office National Interprofessionnel des Oléagineux et des Plantes Textiles (O.N.I.O.L.). 


\section{Références}

1. Mathieu J.P., Fournier G. La sélection du chanvre en France. Psychotropes $1996 ; 2$ (1) : 53-60.

2. Fournier $\mathrm{G}$. La sélection du chanvre à fibres (Cannabis sativa L.) en France. chanvre et THC. C. R. Acad. Agric. Fr. $2000 ; 86(7): 209-17$.

3. Fournier G., Beherec O., Bertucelli S., Mathieu J.P. A propos des conditions d'échantillonnage pour le dosage du delta-9-tétrahydrocannabinol dans les variétés de chanvre à usage industriel. Ann. Toxicol. Anal. 2001 ; $13: 275-81$.

4. Fournier G. A propos de la teneur en delta-9-THC dans les variétés de chanvre à fibres cultivées en France. Ann. Toxicol. Anal. $2003 ; 15: 30-4$.

5. Code de la Santé Publique - Article R. 5181 - Décret $n^{\circ}$ 88-1231 du 29 décembre 1988, art. 1er J.O. du 31 décembre 1988.

6. Code de la Santé Publique - Arrêté du 22 août 1990 portant application de l'article R. 5181 J.O. du 4 octobre 1990.

7. Code de la Santé Publique - Arrêté du 27 mai 1997 modifiant l'arrêté du 22 août 1990 portant application de l'article R. 5181 du code de la santé publique pour le cannabis - J.O. du 31 mai 1997.

8. Code de la Santé Publique - Arrêté du 2 juillet 1999 modifiant l'arrêté du 22 août 1990 portant application de l'article R. 5181 du code de la santé publique pour le cannabis - J.O. du 8 juillet 1999.

9. Journal officiel des Communautés européennes Règlement (CE) $n^{\circ} 1177 / 2000$ de la Commission du 31 mai 2000 modifiant le règlement $(\mathrm{CEE}) \mathrm{n}^{\circ} 1164 / 89$ relatif aux modalités d'aide concernant le lin textile et le chanvre.
10. Journal officiel des Communautés européennes Règlement (CE) $n^{\circ}$ 1672/2000 de la Conseil du 27 juillet 2000 modifiant le règlement (CE) $n^{\circ} 1251 / 1999$ instituant un régime de soutien aux producteurs de certaines cultures arables, pour y inclure le lin et le chanvre destinés à la production de fibres.

11. Journal officiel des Communautés européennes Règlement (CE) $n^{\circ}$ 2860/2000 de la Commission du 27 décembre 2000 modifiant le règlement (CE) $\mathrm{n}^{\circ}$ 2316/1999 portant modalités d'application du règlement (CE) $n^{\circ} 1251 / 1999$ du Conseil instituant un régime de soutien aux producteurs de certaines cultures arables pour y inclure le lin et le chanvre destinés à la production de fibres, préciser les règles concernant les superficies gelées et modifier les superficies de base pour la Grèce et le Portugal.

12. Journal officiel des Communautés européennes Règlement (CE) $n^{\circ} 327 / 2002$ de la Commission du 21 février 2002 modifiant le règlement (CEE) $n^{\circ}$ 2316/1999 portant modalités d'application du règlement $(C E) \mathrm{n}^{\circ}$ 1251/1999 du Conseil instituant un régime de soutien aux producteurs de certaines cultures arables.

13. Journal officiel des Communautés européennes Règlement (CE) $n^{\circ} 335 / 2003$ de la Commission du 21 février 2003 modifiant le règlement (CEE) $n^{\circ} 2316 / 1999$ portant modalités d'application du règlement $(\mathrm{CE}) \mathrm{n}^{\circ}$ 1251/1999 du Conseil instituant un régime de soutien aux producteurs de certaines cultures arables.

Le Code de la Santé Publique peut être retrouvé sur le site : http://www.legifrance.gouv.fr

Le Journal officiel des Communautés européennes peut être retrouvé sur le site : http://europa.eu.int/eur-lex/fr/archive/index.html 